\title{
Effects of the Collimator Magnification Factor in the Geometrical Calibration of SPECT Systems
}

\author{
Debora Salvado, Student Member, IEEE, Kjell Erlandsson, and Brian F. Hutton, Senior Member, IEEE
}

\begin{abstract}
In compact systems, precise measurement in the projection space may be compromised due to minification. The objective of this work is to investigate the impact of the magnification factor in a model-based calibration procedure. This has direct relevance to the geometrical calibration of the clinical INSERT camera.

Projection data from three point sources were simulated for a single pinhole collimator with magnification and single pinhole and slit-slat collimators with minification, for 100 noise realizations and 3 count levels. Model-based calibration was performed to estimate geometric parameters and data corresponding to a Derenzo phantom were simulated and reconstructed with true and worst-case estimates for each collimator. Experimental projection data were acquired with an INSERT prototype camera and four ${ }^{99 m}$ Te line sources in different locations within the FoV. The collimator-CoR distance was varied in order to obtain different minifications and model-based calibration was performed.

The results from the simulations suggest that calibration is less robust when minification is present, with higher biased calibration parameters, which result in activity underestimation. For experimental data, estimated parameters improved with a higher magnification factor, in line with the simulation results. However, some inconsistencies in the results suggest that there is still room for improvements.

To conclude, geometric calibration of SPECT systems is more sensitive to minification than magnification, which will impact image quality.
\end{abstract}

Index Terms-geometrical calibration, minification, SPECT/MR.

\section{INTRODUCTION}

$\mathbf{P}$ RECISE geometric calibration of SPECT cameras is essential to obtain a good reconstructed image [1]. Calibration can be achieved by measuring directly the system matrix, scanning a point source through the whole field of view (FoV), with enough counts to obtain the point spread function (PSF) for each aperture of the collimator [2]. Variations of this method include measuring the PSF in a limited number of points that samples the FoV and interpolating for the remaining positions [3], [4]. These methods are highly accurate and well-suited for stationary systems. However, the duration of the scanning process and the need for sophisticated positioning

Manuscript received December 6, 2016. This work was supported by a PhD Fellowship SFRH/BD/88093/2012 from the Fundação para a Ciência e Tecnologia (FCT), Portugal, co-sponsored by the INSERT collaboration, which is supported by the EC under the FP7-HEALTH program (305311), and by the National Institute for Health Research, University College London Hospitals Biomedical Research Centre.

All authors are with the Institute of Nuclear Medicine, University College London, UK. B. F. Hutton is also with the Centre for Medical Radiation Physics, Univ. Wollongong, Australia.

Corresponding author: D. Salvado, debora.salvado.12@ucl.ac.uk.
TABLE I

GEOMETRICAL CALIBRATION PARAMETERS.

\begin{tabular}{c|l} 
Param. & Description \\
\hline$f$ & Focal length \\
$d$ & Focal point to centre of rotation distance \\
$m$ & Mechanical offset \\
$\phi$ & Tilt between detector and rotation axis \\
$\psi$ & Twist of the pixel grid in relation to detector \\
$e_{u}$ & Electrical shift in transverse direction $\vec{u}$ \\
$e_{v}$ & Electrical shift in axial direction $\vec{v}$
\end{tabular}

tools, that might not be compatible with the magnetic field of the MR, make them impractical in a clinical setting.

The alternative method is to model the system matrix as a function of geometric parameters. It has been shown that a pinhole aperture can be fully described by seven parameters [5], and that geometric calibration can be achieved by minimizing the square distances between estimated and measured projections of at least three non-collinear point sources. This method is well suited for standard pinhole SPECT cameras that benefit from the magnification of a small FoV [6].

With the latest advances in detector technology and the need for compact systems - e.g. the INSERT SPECT/MRI system [7], [8], small cameras can be used to image large FoVs, trading off minification with high intrinsic resolution to achieve a system resolution similar to that of a standard SPECT system. However, with minification, precise measurement in the projection space, required for calibration purposes, may be compromised. The objective of this study is to investigate the impact of the magnification factor in a model-based calibration procedure, which has direct relevance to the geometrical calibration of the clinical INSERT camera.

\section{Materials AND Methods}

\section{A. Simulations}

Seven calibration parameters (Table I) were defined for a single pinhole collimator with magnification factor $M$ of 4 and $0.25\left(\mathrm{PH}_{\mathrm{mag}}\right.$ and $\left.\mathrm{PH}_{\mathrm{min}}\right)$. The detector size, FoV and intrinsic resolution were matched accordingly: $20 \mathrm{~cm}, 5 \mathrm{~cm}$ and $3 \mathrm{~mm}$ for the magnification case, and $5 \mathrm{~cm}, 20 \mathrm{~cm}$ and $1 \mathrm{~mm}$ for the minification case. Simulated data were generated from ideal projections of three non-collinear point sources, blurred according to the system resolution and the parallax effects. Data were then scaled for different count levels and Poisson noise added. This procedure was repeated for 100 noise realizations and three count levels: $10^{3}, 10^{5}$ and $10^{7}$. The geometric calibration parameters were estimated using a constrained nonlinear optimization algorithm in Matlab (The 


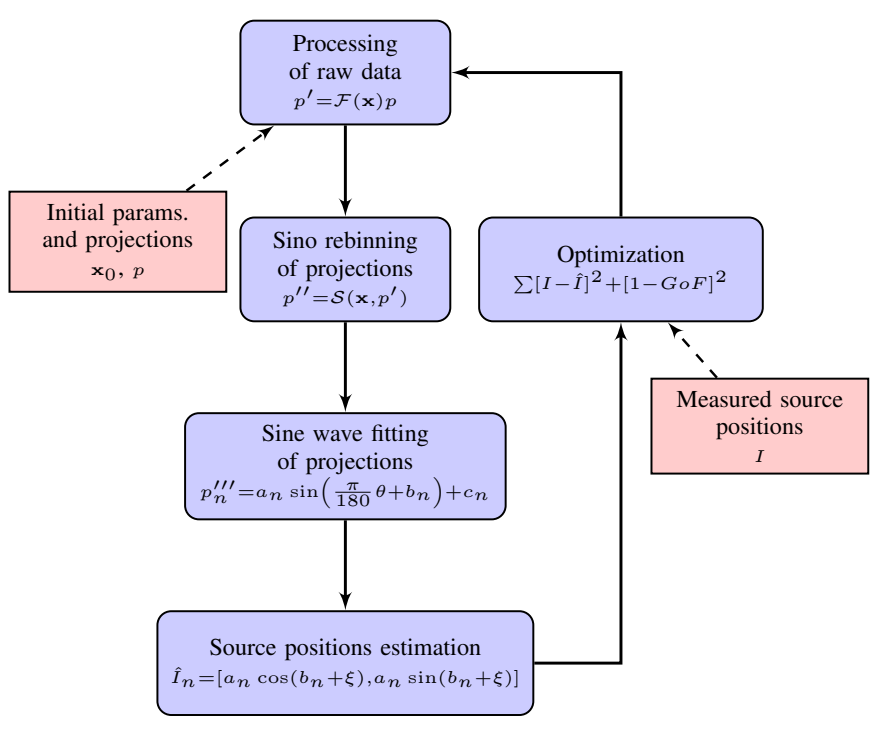

Fig. 1. Framework of the applied model-based geometrical calibration method. $\mathbf{x}$ corresponds to the set of geometrical parameters $\left[f, d, m, \phi, \psi, e_{u}, e_{v}\right], p$ to the projection data, and GoF to the sine-wave goodness of fit of Sino-rebinned projection data.

Mathworks, Inc., Natick, MA, USA), that minimises the sum of the square distances between true and simulated projection data from the three point sources [5]. Bias and standard deviation (SD) of the parameter estimates were obtained for each calibration parameter.

For a slit-slat collimator, the calibration problem in the transaxial direction is similar to that of a pinhole system, but we assume the number of parameters is reduced to five for the slit component: $f, d, m, \phi$ and $e_{u}$. These geometrical parameters were evaluated for a single slit-slat collimator with minification $\left(\mathrm{SS}_{\min }, M=0.25\right)$, with the same projection and calibration procedure described previously, and repeated for 100 noise realizations and three count levels.

For each collimator, $\mathrm{PH}_{\mathrm{mag}}, \mathrm{PH}_{\min }$ and $\mathrm{SS}_{\min }$, the set of geometric calibration parameters with highest deviation from the true parameters was identified from the two lower noise datasets, and used to reconstruct simulated data corresponding to a Derenzo hot-rod phantom. The diameter of the rods in the phantom were 7-12 $\mathrm{mm}$. Reconstruction was also performed with the true calibration parameters. Profiles along $\mathrm{x}$ and $\mathrm{y}$ directions of the reconstructed images were obtained for each collimator and calibration case.

\section{B. Measurements}

Projection data were acquired for 30 angles covering $360^{\circ}$ with an INSERT prototype detector [7] of size $5 \times 5 \mathrm{~cm}$, a Mini-Slit-Slat (MSS) collimator [8] and four ${ }^{99 m} \mathrm{Tc}$ line sources placed on a rotating stage at different radial locations within the FoV: $69.00,51.75,34.50$ and $17.25 \mathrm{~mm}$ from the centre of rotation $(\mathrm{CoR})$ at $90^{\circ}$ intervals. The procedure was repeated for three distances from the collimator to the CoR: $165.00 \mathrm{~mm}, 106.78 \mathrm{~mm}$, and $48.57 \mathrm{~mm}$ (Figure 2), adjusting the radial positions accordingly, in order to get different magnification factors. Model-based geometric calibration was performed as described in Figure 1: raw projection data $p$

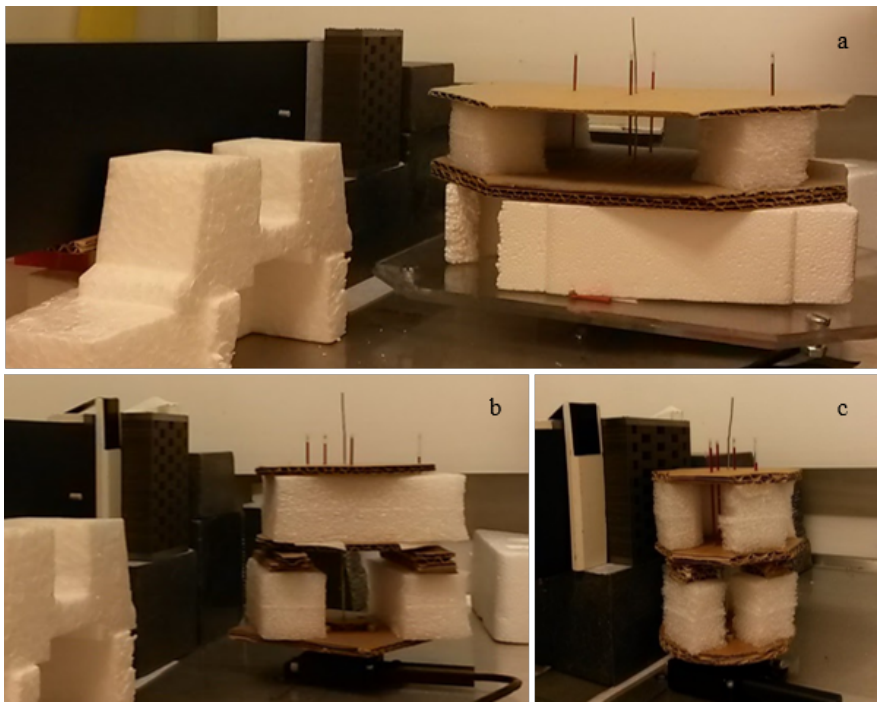

Fig. 2. Experimental setup: detector (black box), MSS collimator and 4-linesource phantom placed on a rotating stage. The distance between collimator

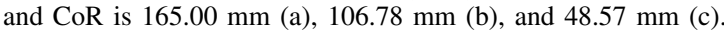

are corrected for detector-shift and sensitivity, and rebinned with the Sino method [9], according to given calibration parameters $\mathbf{x}$; each curve is then fitted with a sine wave, and the fitted parameters used to estimate the corresponding source position; optimization of the calibration parameters is achieved by minimising the sum of the squared distances between true $I$ and estimated $\hat{I}$ source positions, with an extra term for the goodness of fit $G o F$ of the sine waves.

\section{RESULTS}

\section{A. Simulations}

For each simulated collimator, Figure 3 shows the estimated geometrical calibration parameters. Comparing results for the two pinhole collimators, $x_{2}, x_{3}$ and $x_{6}$ parameters show higher relative bias and $\mathrm{SD}$ for $\mathrm{PH}_{\min }$ than $\mathrm{PH}_{\mathrm{mag}}$, while for $x_{1}$ the reverse is true. Comparing the estimates for the collimators with minification, $\mathrm{SS}_{\min }$ parameters show lower relative bias than $\mathrm{PH}_{\min }$, but higher SD. For all the simulated collimators, $\mathrm{SD}$ is reduced for a higher number of counts, i.e. lower noise.

Taking the worst-case calibration scenario of each collimator at the two lower noise levels, Figure 4 shows profiles along the $\mathrm{x}$ and $\mathrm{y}$ direction of the reconstructed Derenzo phantom, together with profiles obtained with the correct calibration parameters. When reconstructed with incorrect compared to correct calibration parameters at the lowest noise level, profiles for the collimators with minification show marginally wider FWHM of the phantom rods and activity underestimation. When increasing the noise, the peak height decreases even more, especially for the $\mathrm{SS}_{\min }$ collimator. No visible difference is observed for the $\mathrm{PH}_{\text {mag }}$ collimator at the two noise levels.

\section{B. Measurements}

Figure 5 shows the measured projection data, one Sinorebinned sinogram and the resulting optimisation plot for experiment 1 (at $165 \mathrm{~mm}$ distance). For the three experiments, 

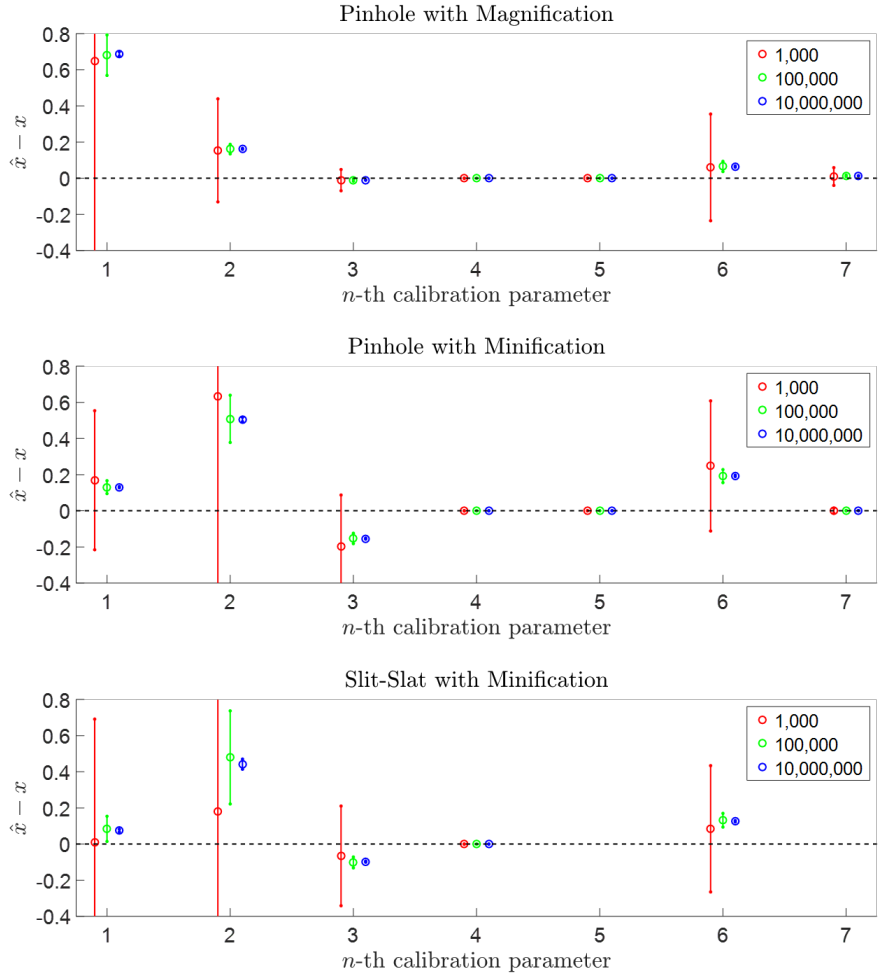

Fig. 3. Relative bias and SD of the estimated calibration parameters for the $\mathrm{PH}_{\text {mag }}, \mathrm{PH}_{\min }$ and $\mathrm{SS}_{\text {min }}$ collimators. Each colour corresponds to a different count level. SD bars of parameters $x_{1}, x_{2}$ and $x_{3}$, and $x_{1}$ and $x_{2}$ exceed the displayed range, top-bottom respectively.

Table II shows the focal length, radius of rotation (RoR), mean line source location errors estimated with the proposed geometric calibration framework and a plot of the source locations within the FoV. The mean error of the estimated line source positions improved with a higher magnification factor, for experiments 2 and 3 compared to 1 .

\section{DISCUSSION}

\section{A. Simulations}

Comparing the $\mathrm{PH}_{\min }$ calibration parameter estimates to the ones from the $\mathrm{PH}_{\text {mag }}$ collimator, the higher bias and SD observed suggests that calibration is less robust when minification is present. When calibrating the $\mathrm{SS}_{\min }$ collimator, the fact that only five parameters are estimated reduces the bias introduced by the minification, however precision is compromised at low counts. For all simulated collimators, the estimated parameters are more stable with higher counts, which corresponds to longer acquisitions.

At the lowest noise level, the profiles of the reconstructed images show that the deviation in the estimated calibration parameters result in small quantitative differences when minification is present, even for the $\mathrm{SS}_{\min }$ collimator, which has low-bias parameter estimates compared to the other collimator geometries. However, when noise increases, activity underestimation becomes problematic with the $\mathrm{SS}_{\min }$ collimator.
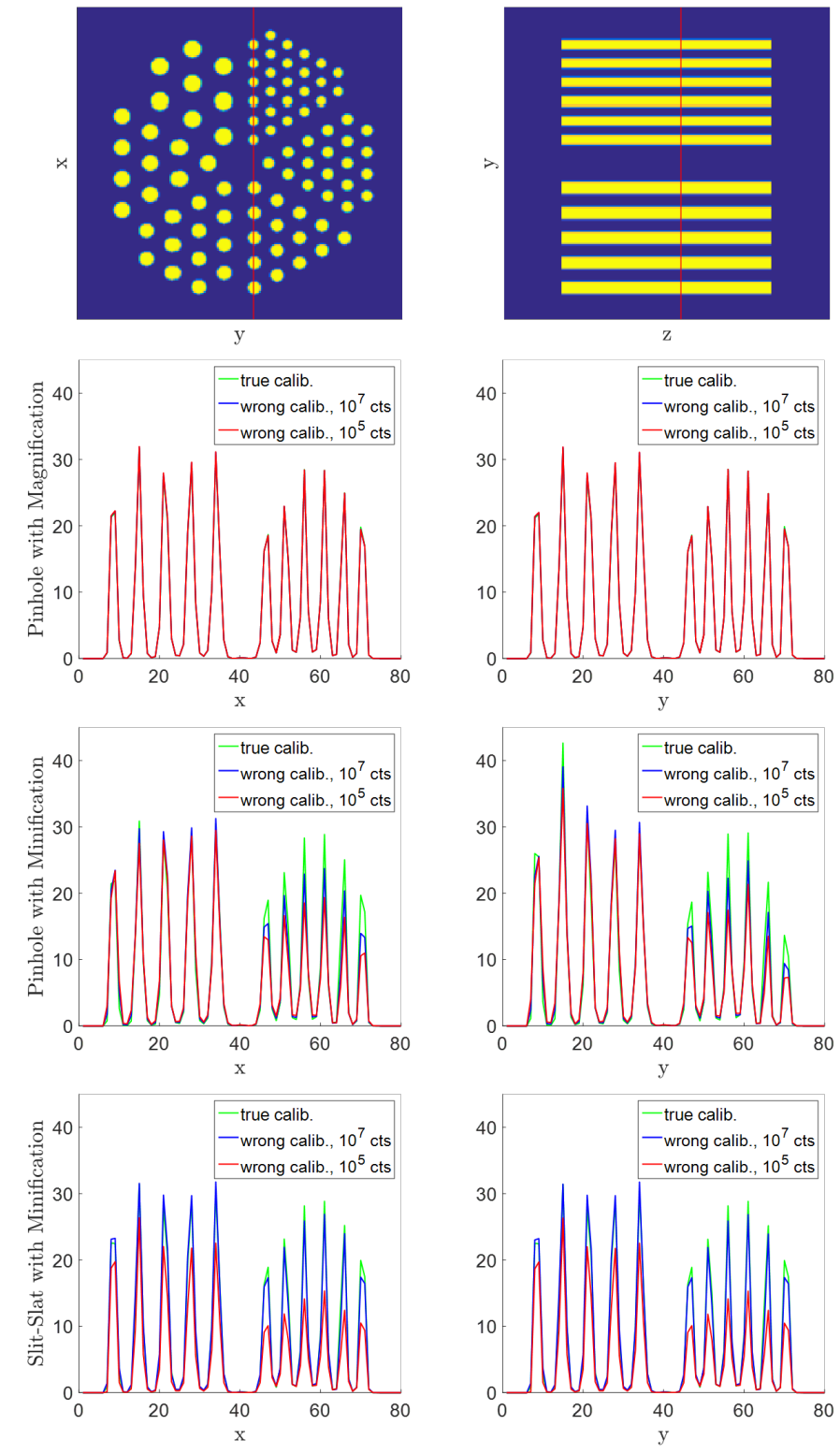

Fig. 4. Derenzo phantom (top) and profiles along $\mathrm{x}$ (left) and $\mathrm{y}$ (right) of the reconstructed images with true (green) and incorrect (blue $-10^{7}$ total counts, red $-10^{5}$ total counts) calibration parameters for the $\mathrm{PH}_{\mathrm{mag}}, \mathrm{PH}_{\min }$ and $\mathrm{SS}_{\mathrm{min}}$ collimators, top-bottom respectively.

\section{B. Measurements}

Results for the experimental data obtained with the prototype INSERT camera are in line with the ones from simulated data, although the mean error of the source positions are slightly higher for experiment 3 compared to 2 . This is due to the fact that sine-fitting of Sino-rebinned projection data are difficult for the source closest to the CoR (flat curve). Regarding the focal length, the estimated values should have been the same across the three experiments, as they were performed with the same collimator. Therefore, the proposed model-based framework needs further improvement in order to properly calibrate the INSERT camera. 

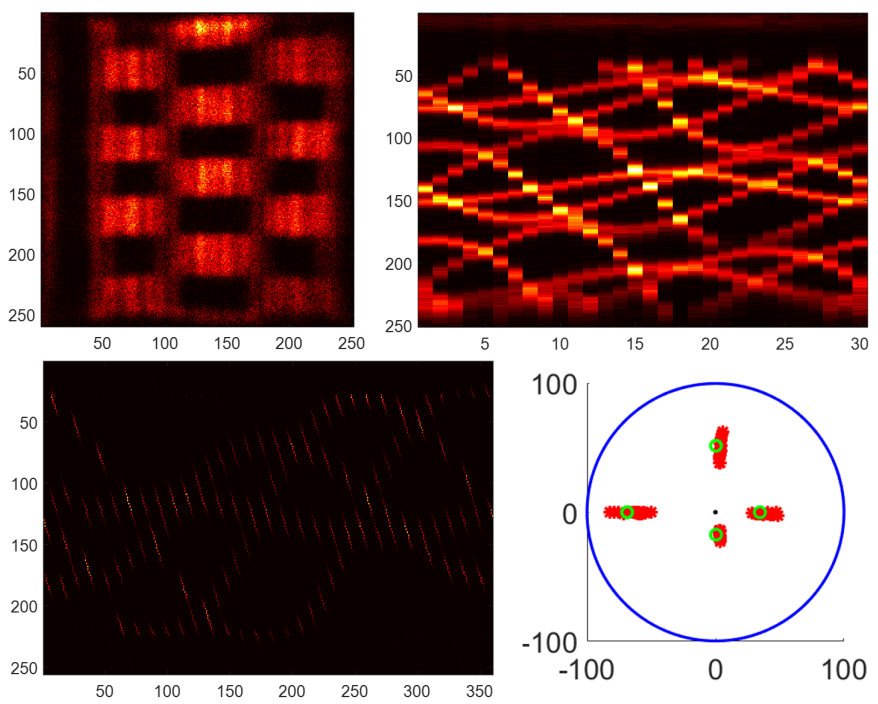

Fig. 5. Planar projection of the measured raw data (top left), sinogram (top right), sinogram after Sino rebinning with initial calibration parameters (bottom left), and plot of the optimization method (bottom right) for the acquisition at a distance of $165 \mathrm{~mm}$. The FoV is represented in blue, the true line source positions in green, and the iteratively estimated positions in red.

TABLE II

EXPERIMENTAL GEOMETRIC PARAMETERS ESTIMATED WITH THE PROPOSED MODEL-BASED CALIBRATION FRAMEWORK. DISTANCE TO COR, FOCAL LENGTH, ROR AND SOURCE POSITION ERRORS SHOWN IN MILLIMETRES.

\begin{tabular}{c|ccc}
$\begin{array}{c}\text { Dist. to } \\
\text { CoR }\end{array}$ & $\mathbf{1 6 5 . 0 0}$ & $\mathbf{1 0 6 . 7 8}$ & $\mathbf{4 8 . 5 7}$ \\
\hline$\hat{f}$ & 24.66 & 23.33 & 21.93 \\
$\frac{R o R}{I-\hat{I}}$ & 166.41 & 124.11 & 68.19 \\
$\frac{1.67}{0 .}$ & 0.83 & 1.03 \\
0.15 & 0.19 \\
&
\end{tabular}

\section{CONCLUSION}

Geometrical calibration of SPECT cameras is more sensitive to minification as opposed to magnification, and requires high precision estimation of the model parameters, in order to avoid deterioration in image quality.

\section{REFERENCES}

[1] J. Nuyts, K. Vunckx, M. Defrise, and C. Vanhove, "Small Animal Imaging with Multi-Pinhole SPECT," Methods, vol. 48, pp. 83-91, 2009.

[2] L. R. Furenlid, D. Wilson, Y. Chen et al., "FastSPECT II: a SecondGeneration High-Resolution Dynamic SPECT Imager," IEEE Transactions on Nuclear Science, vol. 51, pp. 631-5, 2004.

[3] F. van der Have, B. Vastenhouw, M. Rentmeester, and F. Beekman, "System Calibration and Statistical Image Reconstruction for Ultra-High Resolution Stationary Pinhole SPECT," IEEE Transactions on Medical Imaging, vol. 27, pp. 960-71, 2008.

[4] B. W. Miller, R. Van Holen, H. H. Barrett, and L. R. Furenlid, "A System Calibration and Fast Iterative Reconstruction Method for Next-Generation SPECT Imagers," IEEE Transactions on Nuclear Science, vol. 59, no. 5, pp. 1990-6, 2012.
[5] D. Beque, J. Nuyts, G. Bormans et al., "Characterization of Acquisition Geometry of Pinhole SPECT," IEEE Transactions on Medical Imaging, vol. 22, pp. 599-612, 2003.

[6] S. Metzler, R. Jaszczak, N. Patil et al., "Molecular Imaging of Small Animals with a Triple-Head SPECT System Using Pinhole Collimation," IEEE Transactions on Medical Imaging, vol. 24, pp. 853-62, 2005.

[7] P. Busca, M. Occhipinti, P. Trigilio et al., "Experimental Evaluation of a SiPM-Based Scintillation Detector for MR-Compatible SPECT Systems," IEEE Transactions on Nuclear Science, vol. 62, no. 5, pp. 2122-2128, Oct 2015.

[8] D. Salvado, K. Erlandsson, A. Bousse et al., "Novel Collimation for Simultaneous SPECT/MRI," in 2014 IEEE Nuclear Science Symposium and Medical Imaging Conference (NSS/MIC), Nov 2014, pp. 1-5.

[9] K. Erlandsson, D. Salvado, and B. F. Hutton, "A Novel Approach to Image Reconstruction and Calibration for a Multi-Slit-Slat SPECT System," in 2016 IEEE Nuclear Science Symposium and Medical Imaging Conference (NSS/MIC), Nov 2016. 